Case Report

\title{
Mesalazine-Induced Myopericarditis in a Patient with a Recent Diagnosis of Crohn's Disease: Apropos of a Case
}

\author{
Michele Sorleto, Stefanie Dürrwald, and Marcus Wiemer \\ Department of Cardiology, Johannes-Wesling-Klinikum Minden, Hans-Nolte-Strasse 1, 32429 Minden, Germany \\ Correspondence should be addressed to Michele Sorleto; michelesorleto@libero.it
}

Received 14 May 2015; Accepted 6 July 2015

Academic Editor: Tayfun Sahin

Copyright (C) 2015 Michele Sorleto et al. This is an open access article distributed under the Creative Commons Attribution License, which permits unrestricted use, distribution, and reproduction in any medium, provided the original work is properly cited.

\begin{abstract}
Mesalazine- (5-aminosalicylic acid-) containing products are a well-known treatment for inflammatory bowel disease, often as first line. Myocarditis is recognized as a very rare possible side effect of this drug treatment. We present a case of mesalazine-induced myopericarditis that was successfully improved by immediate cessation of the medication.
\end{abstract}

\section{Introduction}

Mesalazine- (5-aminosalicylic acid-) containing products are a well-known treatment for inflammatory bowel disease, often as first line. Hypersensitive reactions to mesalazine have been reported and include mostly gastrointestinal upset and headaches [1]. Although the prevalence is very rare, myocarditis is recognized as a possible side effect of these medications [2-4].

\section{Case Presentation}

Here, we present a case of mesalazine-induced myopericarditis in an 18-year-old male patient with a recent diagnosis of Crohn's disease on treatment with mesalazine and low dosages of prednisone. The young patient was admitted to our hospital via emergency room with retrosternal chest pain spreading to the left arm for several hours. The inflammatory bowel disease was well controlled and there was no reported infection in the last 6 months. The physical examination was normal and there were no signs of hemodynamic instability or heart failure. Initial 12-lead electrocardiogram showed a sinus rhythm and ST-segment elevation with upward concavity in leads II, III, aVF, and V4-V6 (Figure 1). The laboratory tests revealed elevated cardiac biomarkers more than 3 times upper limits (peak troponin hs $1158 \mathrm{pg} / \mathrm{mL}$ and peak total Creatin kinase $547 \mathrm{U} / \mathrm{L}$ ), a C-reactive protein concentration of $97.4 \mathrm{mg} / \mathrm{L}$, and other blood tests within normal parameters (Table 1). A coronary angiography was performed and demonstrated normal epicardial vessels. A cardiovascular magnetic resonance imaging confirmed the coronary angiography result and also there was no hint for a myocardial infarction. However, it showed a late gadolinium enhancement and a myopericardial oedema. As myopericarditis was suspected as an adverse reaction associated with treatment with mesalazine, the drug was immediately stopped with maintaining low dosages of prednisone and metamizol. After that, the retrosternal chest pain has gone and the cardiac biomarkers decreased to normal value. The abnormal ECG findings have been gradually normalized within 7 days. Echocardiographic measurements were within the normal limits. After 8 days the patient was discharged in a good condition to home.

\section{Discussion}

Cardiac involvement can be associated with Crohn's disease as an extraintestinal manifestation of the inflammatory bowel disease. It has also been described as a side effect of the treatment of inflammatory bowel disease with mesalazine. Myocarditis is recognized as a very rare possible side effect of mesalazine- (5-aminosalicylic acid-) containing products, generally occurring 2-4 weeks after the initial exposure to the drug. Several cases were described in the literature. Resolution of symptoms has occurred in all reported cases within one week after drug discontinuation [5-8]. 


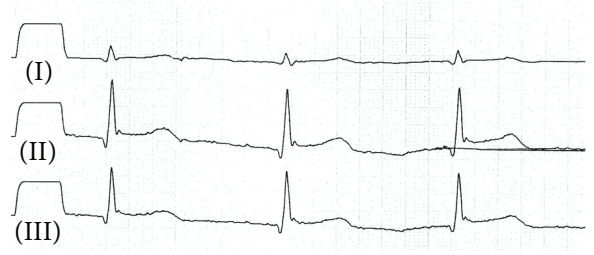

(a)

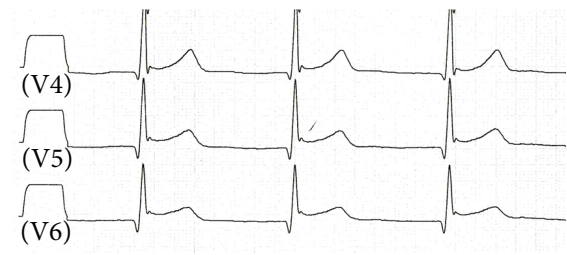

(b)

FIGURE 1: Initial 12-lead electrocardiogram: sinus rhythm and ST-segment elevation with upward concavity in leads II, III, aVF, and V4-V6.

TABLE 1: Initial and follow-up biochemistry values.

\begin{tabular}{lcccc}
\hline $\begin{array}{l}\text { Laboratory chemical } \\
\text { analysis }\end{array}$ & $\begin{array}{c}\text { Day 1 } \\
4: 50\end{array}$ & $\begin{array}{c}\text { Day 1 } \\
7: 40\end{array}$ & $\begin{array}{c}\text { Day 4 } \\
9: 30\end{array}$ & $\begin{array}{c}\text { Day 7 } \\
9: 30\end{array}$ \\
\hline Troponin hs (pg/mL) & 822.6 & 1158.0 & 149.3 & 9.3 \\
Creatin kinase (U/L) & 432 & 547 & 43 & 39 \\
C-reactive protein (mg/L) & 97.4 & & 58.2 & 11.1 \\
Leukocyte (G/L) & 14.7 & & 12.1 & 8.0 \\
\hline
\end{tabular}

Although the precise pathophysiology of mesalazine-induced myopericarditis is poorly understood, it is thought to be a hypersensitivity reaction rather than a cytotoxic effect. A proposed mechanism is humoral-mediated hypersensitivity in which antibodies formed against mesalamine cross-react with cardiac tissue causing inflammation [9]. An eosinophilic infiltration of the myocardium on endomyocardial biopsy has been described, which seems to confirm the link between mesalazine and hypersensitivity [10]. In our case the patient has been treated for 6 weeks with mesalazine containing oral medication, before the drug induced cardiovascular toxicity. Even though myopericarditis is an uncommon adverse reaction to mesalazine, it seemed to be attributed in our young patient during drug treatment. Within 48 hours after discontinuation of the medication, the sick feeling person evolved well and remained asymptomatic, with decreasing cardiac biomarkers. Considering this clinical course plus the fact that there was no previous infection, the immediate drug withdrawal achieved clinical improvement. Therefore, myopericarditis was considered to be the most likely diagnosis. In conclusion, we present a case of mesalazine-induced myopericarditis that was successfully improved by immediate cessation of the medication. In our case, the onset and resolution of symptoms within one week after drug discontinuation were similar to those reported in literature $[3,4,7,8]$. This case identifies the importance of prompt evaluation, diagnosis, and eliciting a thorough medical history when new medications are started. The risk of mesalamine-induced cardiac involvement needs to be considered in patients with inflammatory bowel disease after recent exposure to the drug. It is suggested that every patient with chest pain during mesalamine therapy should be evaluated via cardiac enzymes, electrocardiogram, echocardiogram, and possibly cardiovascular magnetic resonance imaging to rule out this rare drug-induced disorder.

\section{Conflict of Interests}

The authors declare that there is no conflict of interests regarding the publication of this paper.

\section{References}

[1] M. L. Scribano, "Adverse events of IBD therapies," Inflammatory Bowel Diseases, vol. 14, supplement 2, pp. S210-S211, 2008.

[2] N. Ishikawa, T. Imamura, K. Nakajima et al., "Acute pericarditis associated with 5-aminosalicylic acid (5-ASA) treatment for severe active ulcerative colitis," Internal Medicine, vol. 40, no. 9, pp. 901-904, 2001.

[3] O. Atay, K. Radhakrishnan, J. Arruda, and R. Wyllie, "Severe chest pain in a pediatric ulcerative colitis patient after 5aminosalicylic acid therapy," World Journal of Gastroenterology, vol. 14, no. 27, pp. 4400-4402, 2008.

[4] L. García-Ferrer, J. Estornell, and V. Palanca, "Myocarditis by mesalazine with cardiac magnetic resonance imaging," European Heart Journal, vol. 30, no. 8, p. 1015, 2009.

[5] C. G. Braga, J. Martins, C. Arantes et al., "Mesalamine-induced myocarditis following diagnosis of Crohn's disease: a case report," Revista Portuguesa de Cardiologia, vol.32, no. 9, pp. 717720, 2013.

[6] T. Sabatini, A. Filippini, F. Nicosia, S. Preti, and M. Bertoli, "Recurrence of myocarditis after mesalazine treatment for ulcerative colitis: a case report," Inflammatory Bowel Diseases, vol. 19, no. 3, pp. E46-E48, 2013.

[7] E. Perez-Colon, G. H. Dadlani, I. Wilmot, and M. Miller, "Mesalamine-induced myocarditis and coronary vasculitis in a pediatric ulcerative colitis patient: a case report," Case Reports in Pediatrics, vol. 2011, Article ID 524364, 5 pages, 2011.

[8] O. Merceron, C. Bailly, A. Khalil et al., "Mesalamine-induced myocarditis," Cardiology Research and Practice, vol. 2010, Article ID 930190, 3 pages, 2010.

[9] R. A. Waite and J. M. Malinowski, "Possible mesalamineinduced pericarditis: case report and literature review," Pharmacotherapy, vol. 22, no. 3, pp. 391-394, 2002.

[10] S. Stelts, M. H. Taylor, J. Nappi, and A. B. Van Bakel, "Mesalamine-associated hypersensitivity myocarditis in ulcerative colitis," Annals of Pharmacotherapy, vol. 42, no. 6, pp. 904-905, 2008. 


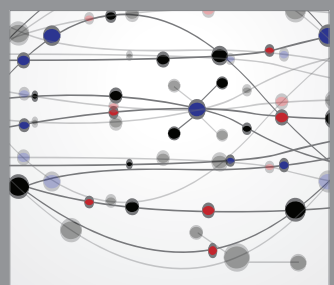

The Scientific World Journal
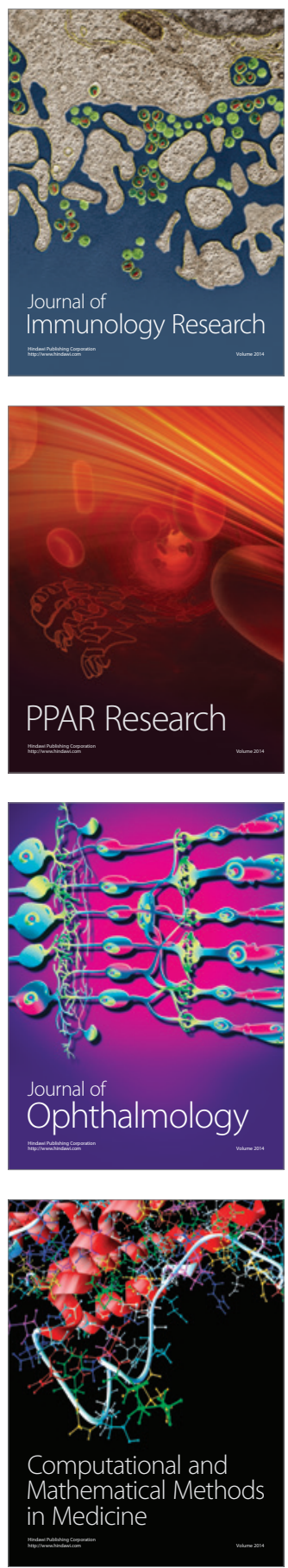

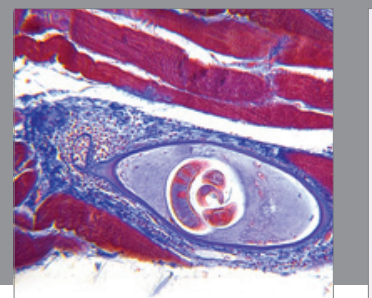

Gastroenterology

Research and Practice
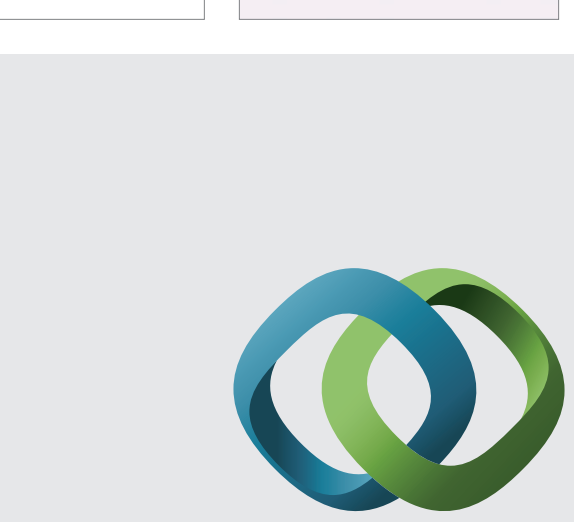

\section{Hindawi}

Submit your manuscripts at

http://www.hindawi.com
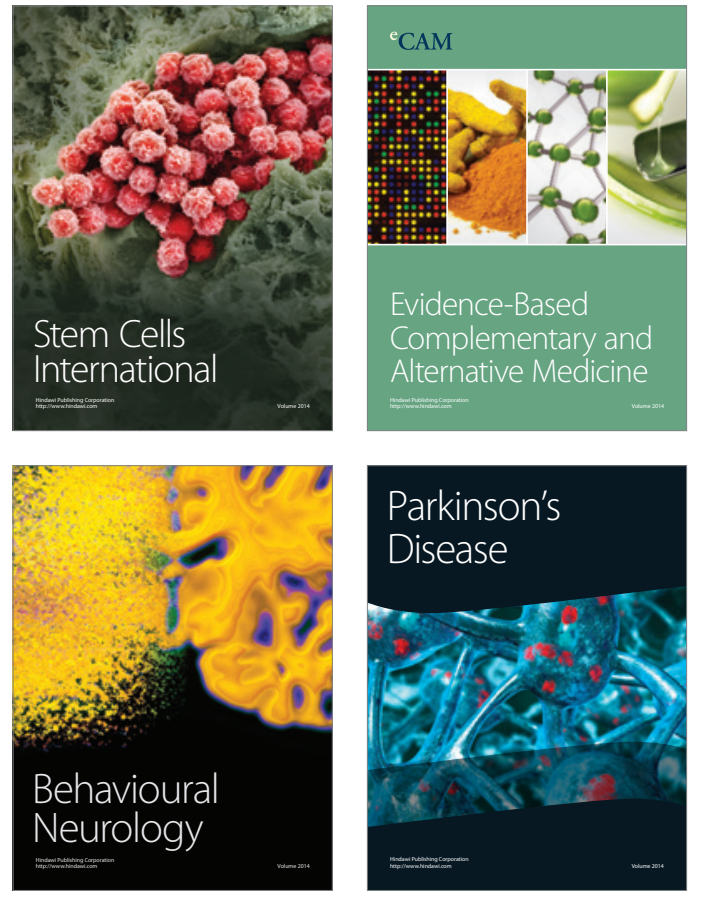
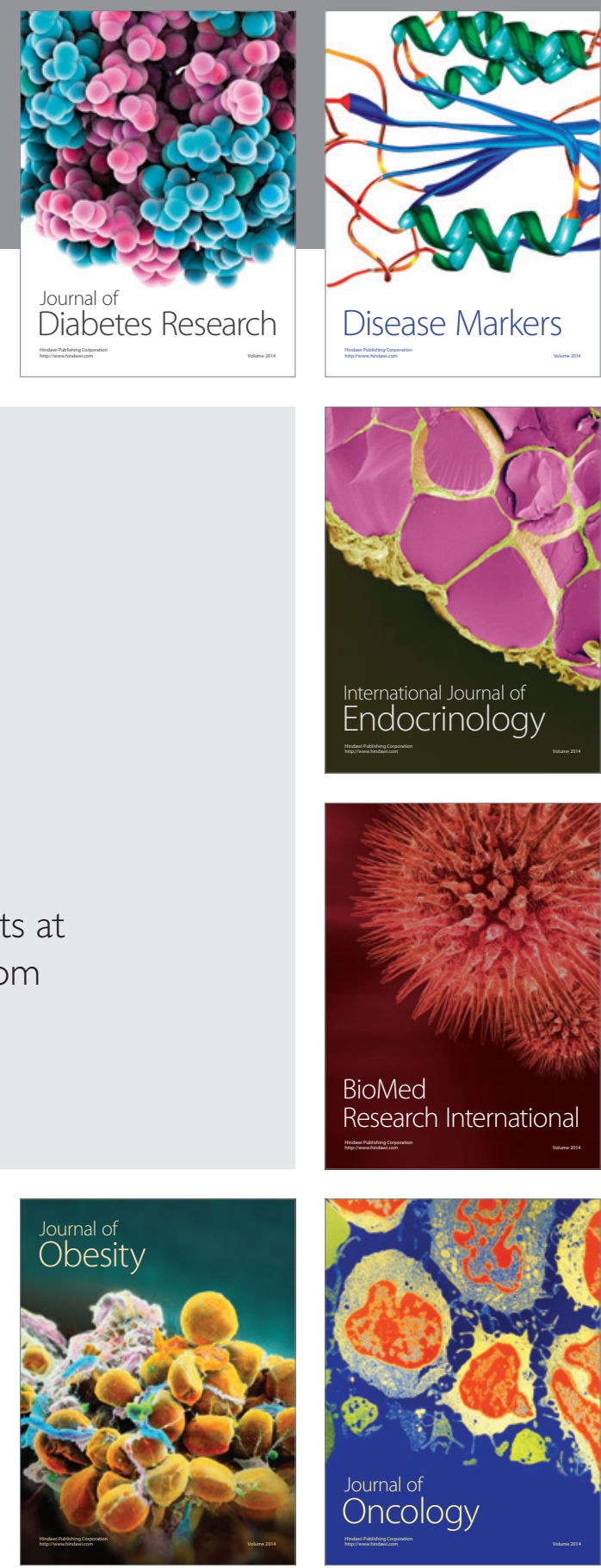

Disease Markers
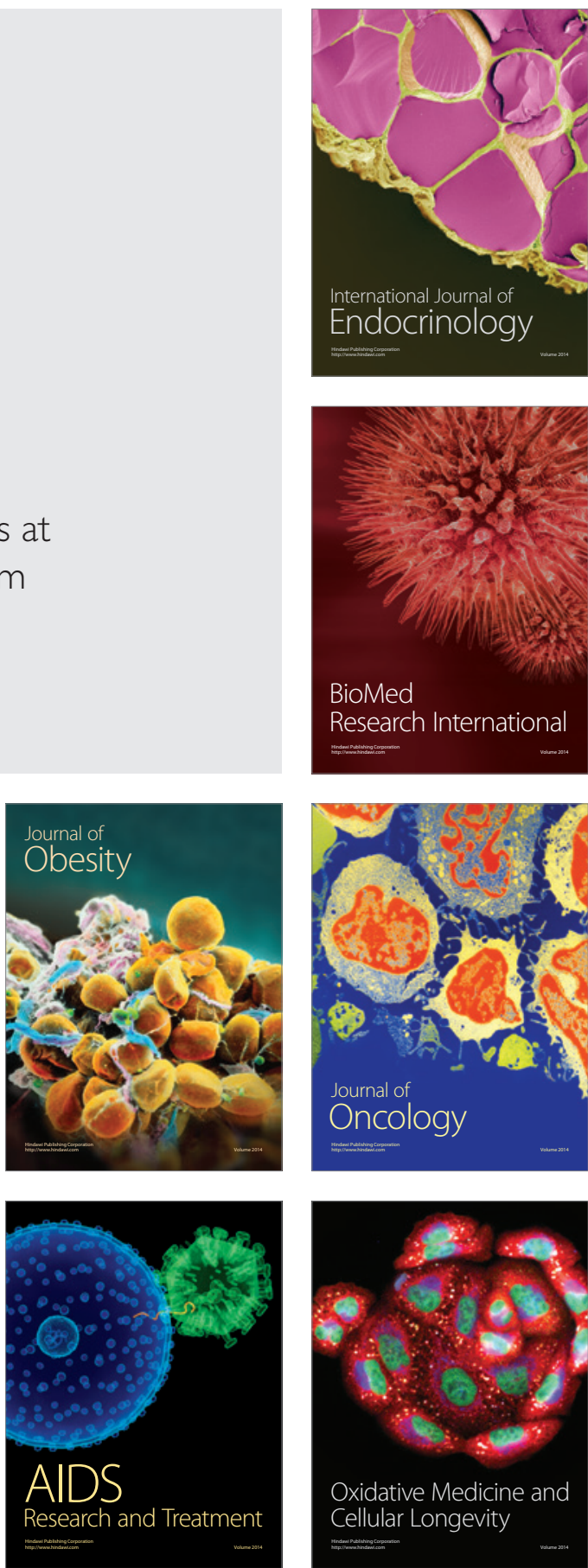